\title{
Glycemic Control, Hand Activity, and Complexity of Biological Signals in Diabetes Mellitus
}

\author{
Hsien-Tsai Wu, ${ }^{1}$ Gen-Min Lin, ${ }^{1,2,3}$ Bagus Haryadi, ${ }^{1,4}$ \\ Chieh-Ming Yang, ${ }^{1}$ and Hsiao-Chiang $\mathrm{Chu}^{1}$ \\ ${ }^{1}$ Department of Electrical Engineering, National Dong Hwa University, Hualien, Taiwan
${ }^{2}$ Department of Medicine, Hualien Armed Forces General Hospital, Hualien, Taiwan
${ }^{3}$ Department of Medicine, Tri-Service General Hospital, National Defense Medical Center, Taipei, Taiwan
${ }^{4}$ Department of Physics, Universitas Ahmad Dahlan, Yogyakarta, Indonesia
}

Correspondence should be addressed to Gen-Min Lin; farmer507@yahoo.com.tw

Received 6 April 2017; Revised 2 July 2017; Accepted 17 August 2017; Published 26 September 2017

Academic Editor: Gaetano Valenza

Copyright (C) 2017 Hsien-Tsai Wu et al. This is an open access article distributed under the Creative Commons Attribution License, which permits unrestricted use, distribution, and reproduction in any medium, provided the original work is properly cited.

\begin{abstract}
Both glycemic control and handgrip strength affect microvascular function. Multiscale entropy (MSE) of photoplethysmographic (PPG) pulse amplitudes may differ by diabetes status and hand activity. Of a middle-to-old aged and right-handed cohort without clinical cardiovascular disease, we controlled age, sex, and weight to select the unaffected (no type 2 diabetes, $n=36$ ), the wellcontrolled diabetes (HbAlc $<8 \%, n=22$ ), and the poorly controlled diabetes (HbAlc $\geq 8 \%, n=22)$ groups. MSEs were calculated from consecutive 1,500 PPG pulse amplitudes of bilateral index fingertips. The small-, medium-, and large-scale MSEs were defined as the average of scale $1\left(\mathrm{MSE}_{1}\right)$, scales 2-4 $\left(\mathrm{MSE}_{2-4}\right)$, and scales 5-10 ( $\left.\mathrm{MSE}_{5-10}\right)$, respectively. Intra- and intergroups were compared by one- and two-sample $t$-tests, respectively. The dominant hand $\mathrm{MSE}_{5-10}$ was lower in the poorly controlled diabetes group than the well-controlled diabetes and the unaffected (1.28 versus 1.52 and $1.56, p=0.019$ and 0.001 , resp.) groups, whereas the nondominant hand $\mathrm{MSE}_{5-10}$ was lower in the well- and poorly controlled diabetes groups than the unaffected group (1.35 and 1.29 versus 1.58 , $p=0.008$ and 0.005 , resp.). The $\mathrm{MSE}_{1}$ of dominant hand was higher than that of nondominant hand in the well-controlled diabetes (1.35 versus $1.10, p=0.048)$. In conclusion, diabetes status and hand dominance may affect the MSE of PPG pulse amplitudes.
\end{abstract}

\section{Introduction}

Poor glycemic control is associated with endothelial dysfunction, an increase of proinflammatory cytokines, and lower adiponectin levels in diabetes [1]. On the contrary, well diabetic control may improve inflammation status and reduce atherogenic low-density lipoprotein concentrations [1, 2]. Numerous observational studies have shown that diabetic microvascular dysfunctions such as cardiac or peripheral autonomic neuropathy are related to physical inactivity and poor glycemic control [3-5]. Furthermore, a meta-analysis of randomized clinical trials revealed that improving glycemic control might reduce the progression of nephropathy and retinopathy in type 2 diabetes [6]. In addition, a combination of resistance and endurance exercise improves vascular endothelial dysfunction and glycemic control, thus leading to a reduction in the prevalence of diabetic microvascular complications [7-9].

Measurement of heart rate variability (HRV) and blood pressure responses to postural change, deep breathing, and Valsalva maneuver has been applied to evaluate the presence of diabetic autonomic neuropathies [10]. Javorka et al. have revealed that many frequency and time domain linear indexes of HRV such as root-mean-square of successive differences of R-R intervals (RMSSD) are significantly reduced in patients with diabetes [11]. Costa et al. proposed the nonlinear index of multiscale entropy (MSE) to reflect the complexity of physiological signals [12], which has been utilized additionally to the linear parameters. In brief, a healthy system exhibits an exquisite complex control to preserve homeostasis, generally leading to a high level of MSE [12]. Silva et al. demonstrated that the nonlinear characteristics of small-time scales MSE 
of HRV are much influenced by parasympathetic activity in experimental mice [13]. By contrast, the nonlinearity of large-time scales MSE are much related to autonomic sympathetic activity and established cardiovascular disease [14]. Previous reports have shown that the MSE of heart rate, blood pressure, and pulse wave velocity might be more sensitive than the linear analysis of HRV parameters to detect subclinical vascular abnormalities in diabetes $[15,16]$.

The magnitude of fingertip photoplethysmographic (PPG) pulse amplitudes is multifactorially influenced by arteriosclerosis, respiratory cycles, cardiac output, blood flow, and pulse pressure [17]. Theoretically the MSE or refined MSE of PPG pulse amplitudes can represent the complexity of cardiovascular and autonomic nervous system network. Since glycemic control and handgrip strength are associated with diabetic microvascular diseases, we hypothesized that the MSE or refined MSE of PPG pulse amplitudes of bilateral fingers and HRV may vary by hand dominance and diabetes status.

\section{Methods}

2.1. Study Population. From July 2009 to October 2010, 113 right-hand dominant men and women, aged between 40 and 80 years, were prospectively recruited at the Ministry of Health and Welfare Hualien Hospital for PPG exams. Of this population, 11 individuals were excluded for a history of heart failure, ischemic stroke, atrial fibrillation, coronary heart disease, peripheral vascular disease, or permanent pacemaker implantation, leaving a study cohort of 102 men and women for the MSE and refined MSE analyses. Diabetes mellitus was defined as glycated hemoglobin (HbAlc) $>6.5 \%$ or fasting plasma glucose $\geq 126 \mathrm{mg} / \mathrm{dl}$ or with hypoglycemic therapy. Of these, 24 individuals had an HbAlc $\geq 8 \%$ (the poorly controlled diabetes group), 29 had an HbAlc < $8 \%$ (the well-controlled diabetes group), and 49 did not have type 2 diabetes (the unaffected group). We matched age, sex, and body mass index at baseline and selected 80 individuals (78.4\%) from the initial study cohort $(22,22$, and 36 from the poorly controlled diabetes, the well-controlled diabetes, and the unaffected groups, resp.) to perform a casecontrol comparison as the MSE might be influenced by these variables. This study has been reviewed and approved by the participating institutions Research Ethics Boards. Each participant signed an informed consent.

2.2. Study Protocol. Medical history, demographic, anthropometric, and laboratory data for the analysis were obtained at the visit in the morning. Measurement of each participant's blood pressure was performed once over left arm in the supine position by an automated oscillometric device (Microlife BP3AG1, Taiwan) after a 20-minute rest. Concentrations of triglycerides and total cholesterol were measured from blood samples obtained after a 12-hour fasting. Diabetic retinopathy signs were identified by a fundus examination. Diabetic nephropathy or chronic kidney disease was diagnosed by a spot urinary albumin-creatinine ratio $\geq 30 \mathrm{mg} / \mathrm{g}$ (microalbuminuria) or an estimated glomerular filtration rate $<90 \mathrm{ml} / \mathrm{min} / 1.73 \mathrm{~m}^{2}$. Peripheral or diabetic neuropathy was evaluated by the presence of numbness, tingling, or pain sensation of upper or lower extremity digits lasting for more than 6 months. Hypertension was defined as having a resting blood pressure $\geq 140 / 90 \mathrm{~mm} \mathrm{Hg}$ or use of antihypertensive medications. Dyslipidemia was defined as high-density lipoprotein cholesterol $<40 \mathrm{mg} / \mathrm{dl}$ in men or $<50 \mathrm{mg} / \mathrm{dl}$ in women, low-density lipoprotein cholesterol $\geq$ $130 \mathrm{mg} / \mathrm{dl}$, or use of lipid-lowering medications.

Theophylline- and caffeine-containing substances were forbidden to use for 12 hours before the visit. In addition, all participants took a rest for 10 minutes or more in a quiet room with a constant temperature at $26 \pm 1^{\circ} \mathrm{C}$ to minimize involuntary vibrations of extremities and constriction of the peripheral vessels, which may lead to erroneous artificial readings from infrared sensors at index fingers. Six-channel electrocardiographic (ECG) pulse wave velocity (PWV) was calculated by an average of acquisition of ECG R waves and simultaneous systolic peak of bilateral index fingertips PPG recordings for 30 minutes $[18,19]$.

2.3. Calculation of Bilateral Hands PPG Pulse Amplitudes Series. To acquire the digital data, the PPG infrared sensors were placed on bilateral index fingertips of each participant. The digitized signals being processed by an analog-to-digital converter (USB-6009 DAQ, National Instruments, Austin, TX, USA) at a frequency of $500 \mathrm{~Hz}$ were analyzed by the Matlab 7.7 software (MathWorks, Massachusetts, USA) and stored on a computer [20]. PPG pulse amplitude was defined as the systolic peak which was presented in Figure 1. In addition, successive 1,500 PPG pulse amplitudes of left and right hands were simultaneously retrieved and shown as $\mathrm{PPGA}_{\mathrm{L}}=\left\{\mathrm{PPGA}_{\mathrm{L}}(1), \mathrm{PPGA}_{\mathrm{L}}(2), \mathrm{PPGA}_{\mathrm{L}}(3), \ldots, \mathrm{PPGA}_{\mathrm{L}}(n)\right\}$ and $\operatorname{PPGA}_{\mathrm{R}}=\left\{\operatorname{PPGA}_{\mathrm{R}}(1), \operatorname{PPGA}_{\mathrm{R}}(2), \operatorname{PPGA}_{\mathrm{R}}(3), \ldots\right.$, $\left.\operatorname{PPGA}_{\mathrm{R}}(n)\right\}$ from each participant.

Due to removing the noises from original PPG data created in nonstationary and nonlinear processes [21], empirical mode decomposition (EMD) method was used [22] to partition the bilateral hands PPGA series that approaches the zero mean. Then the PPGA series were normalized as shown in (1). In (1), $\mathrm{SD}_{\mathrm{PPGA}_{\mathrm{L}}}$ and $\mathrm{SD}_{\mathrm{PPGA}_{\mathrm{R}}}$ were the standard deviations of left and right hand PPGA series, respectively. $\overline{\mathrm{PPGA}_{\mathrm{L}}}$ and $\overline{\mathrm{PPGA}_{\mathrm{R}}}$ represented the means of left and right hand PPGA series, respectively. The MSE and refined MSE were calculated on the normalized results, $n \mathrm{PPGA}_{\mathrm{L}}(i)$ and $n \operatorname{PPGA}_{\mathrm{R}}(j)$ :

$$
\begin{aligned}
n \operatorname{PPGA}_{\mathrm{L}}(i) & =\frac{\operatorname{PPGA}_{\mathrm{L}}(i)-\overline{\mathrm{PPGA}_{\mathrm{L}}}}{\mathrm{SD}_{\mathrm{PPGA}_{\mathrm{L}}}} \\
n \mathrm{PPGA}_{\mathrm{R}}(j) & =\frac{\operatorname{PPGA}_{\mathrm{R}}(j)-\overline{\mathrm{PPGA}_{\mathrm{R}}}}{\mathrm{SD}_{\mathrm{PPGA}_{\mathrm{R}}}}
\end{aligned}
$$

2.4. MSE Analysis of Bilateral Hands PPG Pulse Amplitudes Series. MSE contains the procedures of coarse-graining and estimation of sample entropy $\left(S_{E}\right)$ for respective coarsegrained time series.

(1) The data series $x(n)$ with length $N$ and the two parameters of $m$ (embedded dimension of the vector) and 

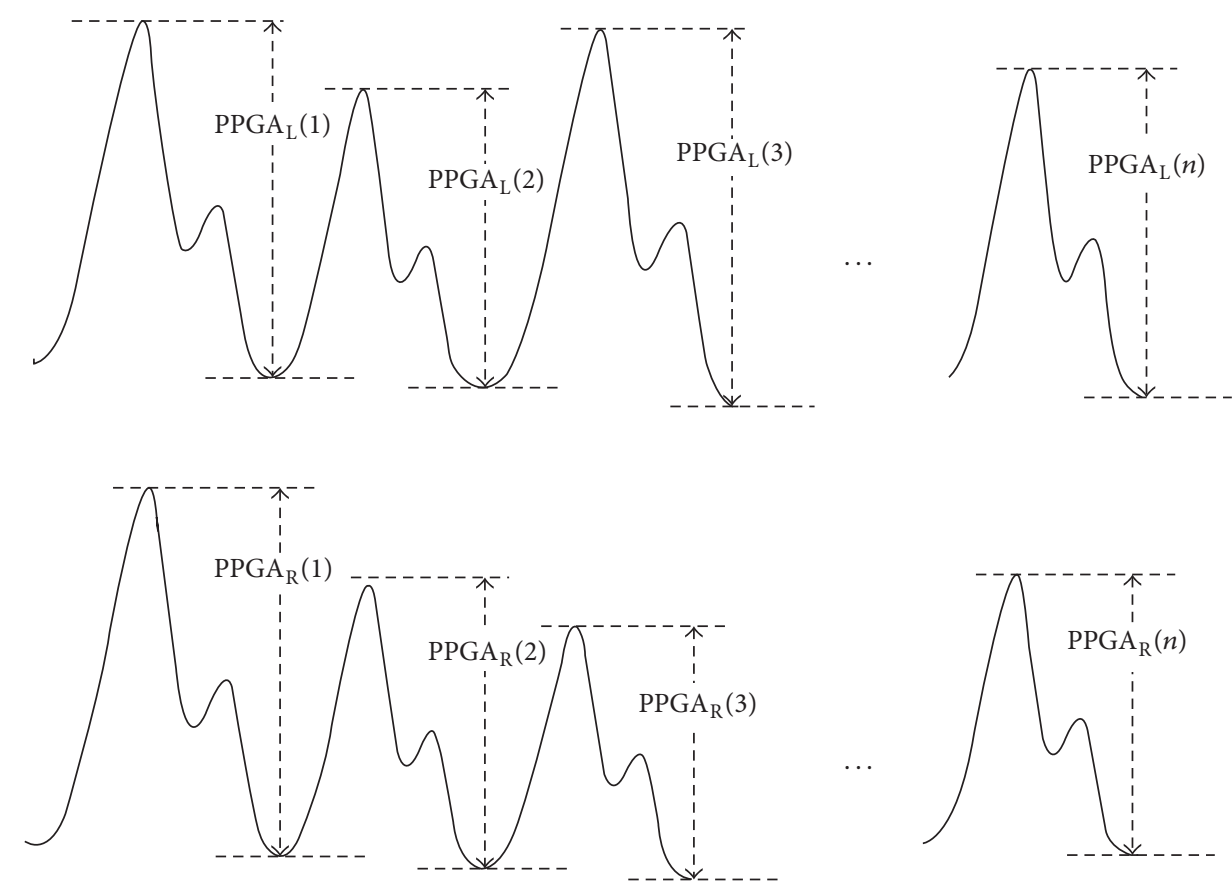

FIGURE 1: Photoplethysmographic pulse amplitudes (PPGA) series of left and right hands (PPGA $\mathrm{L}_{\mathrm{L}}$ and PPGA $\mathrm{R}_{\mathrm{R}}$, resp.) were defined as the distance between peak and triumph of each pulse waveform and simultaneously acquired from signal 1 to signal 1,500.

$r$ (tolerance) are defined. $N=1,500$ for $n$ PPGA $(i)$ and $n \mathrm{PPGA}_{\mathrm{R}}(j)$, respectively. We set $m=2$ and $r=$ $0.15 \times \mathrm{SD}_{\mathrm{PPGA}}$ based on previous Costa et al's study recommendation [14].

(2) $N-m+1$ vectors, each of size $m$, are defined and composed as follows:

$u_{m}(i)=\left\{x_{i}, x_{i+1}, \ldots, x_{i+m-1}\right\}, \quad 1 \leq i \leq N-m+1$.

(3) $d\left[u_{m}(i), u_{m}(j)\right]$ is defined as the maximum value: $d\left[u_{m}(i), u_{m}(j)\right]=\max \left\{\left|x_{i+k}-x_{j+k}\right|: 0 \leq k \leq\right.$ $m-1\}(i \neq j)$. The number of $d\left[u_{m}(i), u_{m}(j)\right]$ within distance $r$ and the ratio of the number to the total $N-m$ for each value of $i \leq N-m+1$ are calculated and an average to all points is defined as

$$
C_{m}(r)=\frac{1}{N-m+1} \sum_{i=1}^{N-m+1} \ln \frac{n_{i}^{m}}{N-m+1} .
$$

(4) The embedded dimension is increased to $m+1$ gives

$$
C_{m+1}(r)=\frac{1}{N-m} \sum_{i=1}^{N-m} \ln \frac{n_{i}^{(m+1)}}{N-m} .
$$

(5) As a result, $S_{E}$ is defined as

$$
S_{E}(m, r, N)=C_{m}(r)-C_{m+1}(r) .
$$

Multiple coarse-grained time series are built up of averaging the data points within nonoverlapping blocks of increasing length, $\tau$ (i.e., the scale factor), as follows:

$$
y_{j}^{(\tau)}=\frac{1}{\tau} \sum_{i=(j-1) \tau+1}^{j \tau} x_{i}, \quad 1 \leq j \leq \frac{N}{\tau} .
$$

Therefore, $S_{E}$ is computed for each new coarse-grained time series $\left\{y_{j}^{(\tau)}\right\}$ of which the length is $N / \tau$ and is plotted as a function of the scale factor [12]. The scale factor $\tau$ up to 10 was selected for a minimal length of the coarse-grained time series equal to 150 beats, a length appropriate for a reliable estimate of $S_{E}$ [23]. As peripheral vasomotor rhythm is typically around 20 seconds, the time scale at $\tau=1$ was defined as small-scale for analyzing PPG pulse amplitudes series using the MSE algorithm [24]. In addition, the time scales, $\tau$ between 2 and 4 , and $\tau$ between 5 and 10 were defined as medium- and large-scales, respectively, based on the study of Bari et al. [24].

2.5. MSE Analysis of ECG $R-R$ Intervals Series. To assess the MSE of ECG R-R intervals series [20], the procedure followed that of PPG pulse amplitudes of bilateral hands. Small-, medium-, and large-scales were defined as scale 1, scales 2-4, and scales 5-10, respectively. The sum of MSE in small-, medium-, and large-scales of series of PPG pulse amplitudes and ECG R-R intervals were averaged and abbreviated as $\mathrm{MSE}_{1}, \mathrm{MSE}_{2-4}$, and $\mathrm{MSE}_{5-10}$, respectively. We set $m=2$ and $r=0.15 \times \mathrm{SD}_{\mathrm{R}-\mathrm{R} \text { intervals }}[14]$.

2.6. Refined MSE Analysis of PPG and ECG Signal Series. The low pass filter's frequency response in MSE calculation poorly manifested by a slow roll-off of the main lobe, aliasing of important side lobes, and a large transition band may not be prevented as the filtered series is downsampled at a rate of one sample every $\tau$ [24]. A refined MSE (RMSE) method proposed by Valencia et al. might improve the flaw of MSE [25]. In brief, the low pass finite impulse response filter in MSE is substituted by a low pass Butterworth filter 
TABLE 1: Baseline characteristics of nondiabetic participants (unaffected), those with well-controlled diabetes (HbAlc $<8 \%$ ), and those with poorly controlled diabetes ( $\mathrm{HbAlc} \geq 8 \%$ ).

\begin{tabular}{|c|c|c|c|}
\hline & Unaffected $(n=36)$ & Diabetes, HbAlc $<8 \%(n=22)$ & Diabetes, HbAlc $\geq 8 \%(n=22)$ \\
\hline Male, \% & $19(52.8)$ & $12(54.6)$ & $12(54.6)$ \\
\hline Age, y & $60.3(8.8)$ & $64.9(6.1)$ & $61.3(7.2)$ \\
\hline Height, m & $161.6(8.4)$ & $162.0(9.2)$ & $161.2(7.5)$ \\
\hline Weight, kg & $68.0(10.1)$ & $71.7(15.7)$ & $71.2(10.4)$ \\
\hline Waist circumstance, $\mathrm{cm}$ & $88.7(8.0)$ & $92.7(13.0)$ & $93.7(10.4)$ \\
\hline $\mathrm{BMI}^{9}, \mathrm{~kg} / \mathrm{m}^{2}$ & $26.0(3.1)$ & $27.2(4.7)$ & $27.5(4.0)$ \\
\hline Pulse rate, beats/min & $73.5(8.6)$ & $77.6(13.8)$ & $83.7(11.4)$ \\
\hline Systolic BP, mmHg & $127.4(18.7)$ & $126.0(21.4)$ & $128.3(16.4)$ \\
\hline Diastolic BP, mmHg & $75.5(10.8)$ & $74.9(11.9)$ & $76.5(9.9)$ \\
\hline Pulse pressure, $\mathrm{mmHg}$ & $51.9(15.7)^{\dagger}$ & $51.1(14.4)$ & $51.8(14.3)$ \\
\hline HbAlc, $\%$ & $6.0(0.4)^{* \dagger}$ & $7.1(0.4)^{\ddagger}$ & $9.6(1.6)$ \\
\hline Fasting glucose, mg/dl & $110.5(24.4)^{* \dagger}$ & $126.0(23.9)^{\ddagger}$ & $189.5(58.4)$ \\
\hline Total cholesterol, mg/dl & $185.5(38.1)$ & $170.9(37.6)$ & $191.2(36.7)$ \\
\hline Triglyceride, mg/dl & $111.1(83.6)^{\dagger}$ & $118.7(47.8)$ & $158.4(76.4)$ \\
\hline HDL-C, mg/dl & $48.7(14.1)$ & $43.8(22.8)^{\ddagger}$ & $41.2(10.9)$ \\
\hline LDL-C, mg/dl & $108.7(29.8)$ & $97.2(23.9)$ & $118.5(29.4)$ \\
\hline Diabetes duration, $\mathrm{y}$ & $0(0)^{* \dagger}$ & $8.2(8.9)$ & $12.5(8.5)$ \\
\hline Hypertension, \% & $15(41.7)^{* \dagger}$ & $16(72.7)$ & $16(72.7)$ \\
\hline Dyslipidemia, \% & $24(66.7)$ & $14(63.6)$ & $17(77.3)$ \\
\hline Peripheral neuropathy, \% & $2(5.6)$ & $3(13.6)$ & $5(22.7)$ \\
\hline Diabetic retinopathy, \% & $0(0)$ & $0(0)$ & $2(9.1)$ \\
\hline Chronic kidney disease, $\%$ & $1(2.8)$ & $1(4.6)$ & $0(0)$ \\
\hline
\end{tabular}

Continuous variables are expressed as mean (standard deviations) and categorical variables as number (percentage). BMI, body mass index; BP, blood pressure; HbAlc, hemoglobin Alc; HDL-C, high-density lipoprotein cholesterol; LDL-C, low-density lipoprotein cholesterol; ${ }^{\mathbf{9}}$ BMI was calculated as body weight $(\mathrm{kg}) /$ square of height $\left(\mathrm{m}^{2}\right){ }^{*} p<0.05$ : diabetes, HbAlc $<8 \%$ versus unaffected; ${ }^{\dagger} p<0.05$ : diabetes, HbAlc $\geq 8 \%$ versus unaffected; ${ }^{\ddagger} p<0.05$ : diabetes, HbAlc $<8 \%$ versus diabetes, HbAlc $\geq 8 \%$.

of order 6 with a cutoff frequency of $0.5 / \tau$ cycles/sample in RMSE [26]. The characteristics of this filter include no side lobes in the stop band, a flat response in the pass band, and a faster roll-off, which limit aliasing efficiently during downsampling. In RMSE, $r$ is set as a percentage of the SD of $\tau$, which could continuously update $r$ with $\tau$ [25].

\section{Statistical Analysis}

Continuous variables were presented as mean (SD) and compared by two-tailed $t$-test. If Kolmogorov-Smirnov test, the normality test, was not fulfilled, Wilcoxon signed rank test was used. Categorical variables were presented as number (percentage) and compared by chi-square test or Fisher's exact test. The PPGA and PWV as well as MSE and RMSE of PPGA and ECG R-R intervals were compared intra- and intergroups by one-sample and two-sample $t$ tests, respectively. Since the PPGA and PWV data were nonnormal distributions, Mann-Whitney $U$ test was used for their intergroups comparison. A 2-tailed value of $p<0.05$ was considered statistically significant. All statistical analyses were performed using SPSS version 14.0 (SPSS Inc., Chicago, IL, USA).

\section{Results}

The baseline characteristics of the age-, sex-, and weightmatched three groups were shown in Table 1. As compared with participants in the unaffected group, those in the diabetes groups seemed to have greater waist size, faster pulse rate, and more proportion of hypertension. As compared with patients with well-controlled diabetes, those with poorly controlled diabetes had higher $\mathrm{HbAlc}$ and more diabetic microvascular complications.

Table 2 shows that the mean PPG pulse amplitudes of bilateral hands did not differ between inter- and intragroups. In contrast, the PWV was higher in both hands of the two diabetes groups, whereas the PWV was similar between two hands in each group. The dominant hand large-scale $\mathrm{MSE}_{5-10}$ was lower in the poorly controlled diabetes group compared with that in the well-controlled diabetes group and the unaffected group (1.28 versus 1.52 and $1.56, p=$ 0.019 and 0.001 , resp.); however, the nondominant hand $\mathrm{MSE}_{5-10}$ was both lower in the poorly and well-controlled diabetes groups than that in the unaffected group (1.29 and 1.35 versus $1.58, p=0.005$ and 0.008 , resp.). The mediumscale $\mathrm{MSE}_{2-4}$ showed a similar trend with the large-scale $\mathrm{MSE}_{5-10}$ in bilateral hands. However, there was no difference in the small-scale $\mathrm{MSE}_{1}$ of bilateral hands between groups. In 
TABLE 2: Comparisons of photoplethysmographic pulse amplitudes, pulse wave velocity, multiscale entropy, and refined multiscale entropy between two hands and between groups.

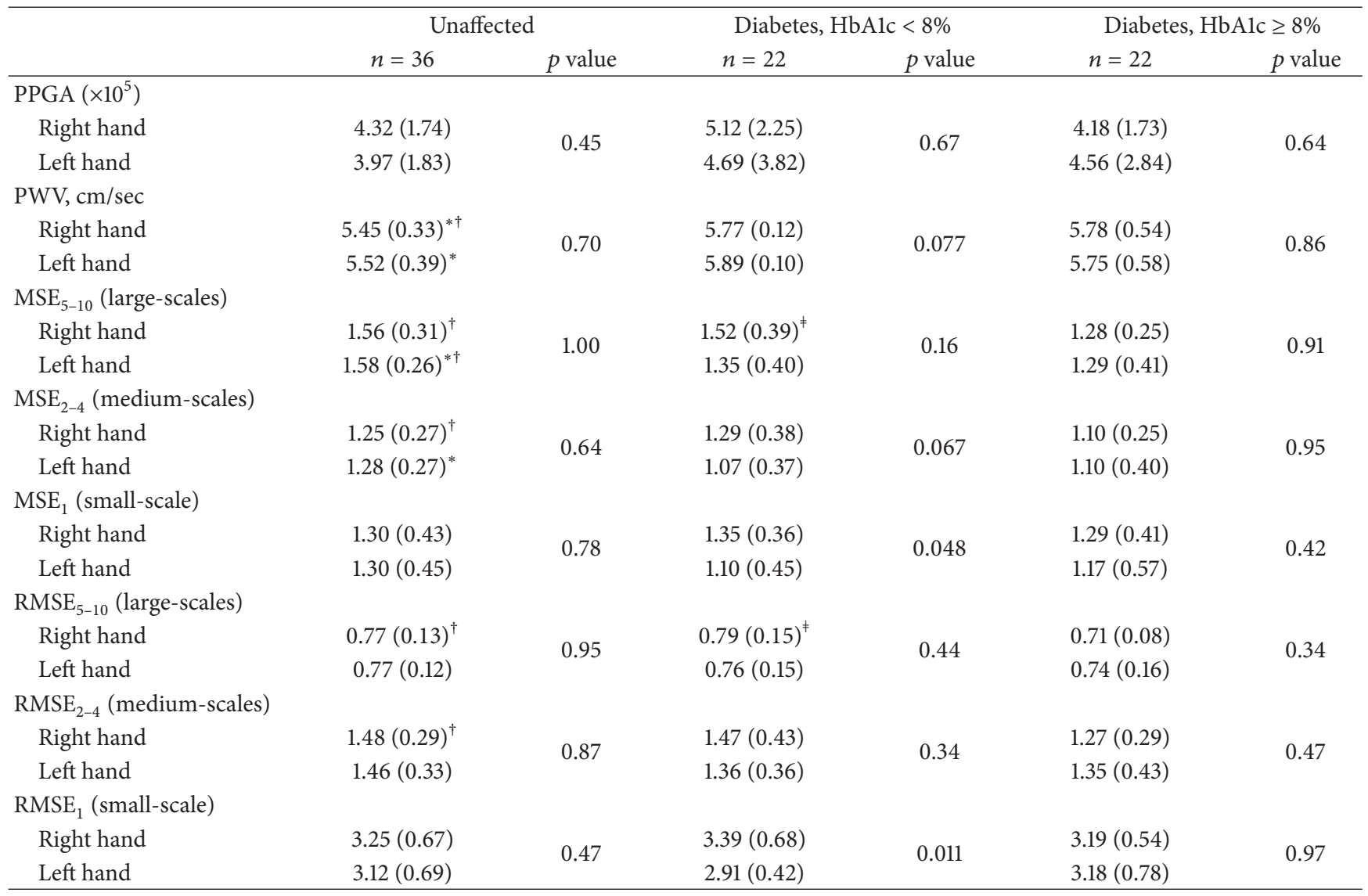

Data are expressed as mean (standard deviations), and one-sample paired $t$-test is used to compare the data between right and left hands shown as $p$ value. MSE, multiscale entropy; PPGA, photoplethysmographic pulse amplitudes; PWV, cardiofingertip pulse wave velocity; RMSE, refined MSE; ${ }^{*} p<0.05$ : diabetes, HbAlc $<8 \%$ versus unaffected; ${ }^{\dagger} p<0.05$ : diabetes, HbAlc $\geq 8 \%$ versus unaffected; ${ }^{\dagger} p<0.05$ : diabetes, HbAlc $<8 \%$ versus diabetes, $\mathrm{HbAlc} \geq 8 \%$.

addition, there were no differences in the medium- and largescale MSE between dominant hand and nondominant hand, while a higher small-scale $\mathrm{MSE}_{1}$ of dominant hand than that of nondominant hand was observed in the well-controlled diabetes group (1.35 versus 1.10, $p=0.048$ ). Notably, the results of RMSE of bilateral hands were consistent with that of MSE in each group.

Figure 2 shows the MSE and RMSE of bilateral hands PPG pulse amplitudes series at each scale factor of the three groups. Generally, the unaffected group had the highest MSE and RMSE levels, and the poorly controlled diabetes group had the lowest MSE and RMSE levels across most scale factors in both left and right hands. For the well-controlled diabetes group, the MSE had similar high level as that of the unaffected group at each scale factor in right dominant hand, whereas the MSE had as low level as that of the poorly controlled diabetes group in left nondominant hand. The pattern of bilateral hands RMSE between each group was consistent with that of MSE.

Figure 3 shows the MSE and RMSE of ECG R-R intervals series at each scale factor of the three groups. Although there was no difference between groups (data not shown), the unaffected group seemed to have high MSE and RMSE levels, and the poorly controlled diabetes group had relatively low MSE and RMSE levels across most scale factors. For the wellcontrolled diabetes group, the MSE had similar high level as that of the poorly controlled diabetes group at each scale factor, but in contrast the RMSE had similar high level as that of the unaffected group.

Supplemental Figures 1 and 2 in Supplementary Material available online at https://doi.org/10.1155/2017/3472121 reveal the MSE and RMSE indexes at each scale factor between two hands of the three groups in the case-control population, respectively.

\section{Discussion}

Our principal findings were that PPG pulse amplitudes rather than ECG R-R intervals for MSE and RMSE analyses were sensitive to evaluate local vascular health. The mediumand large-scale MSE or RMSE index were found higher in participants without diabetes (more complex PPG pulse amplitudes) than those in participants with poorly controlled diabetes. Moreover, greater handgrip strength (dominant hand) might increase the small-scale MSE ${ }_{1}$ or RMSE $E_{1}$ index of PPG pulse amplitudes with euglycemia status. However, 


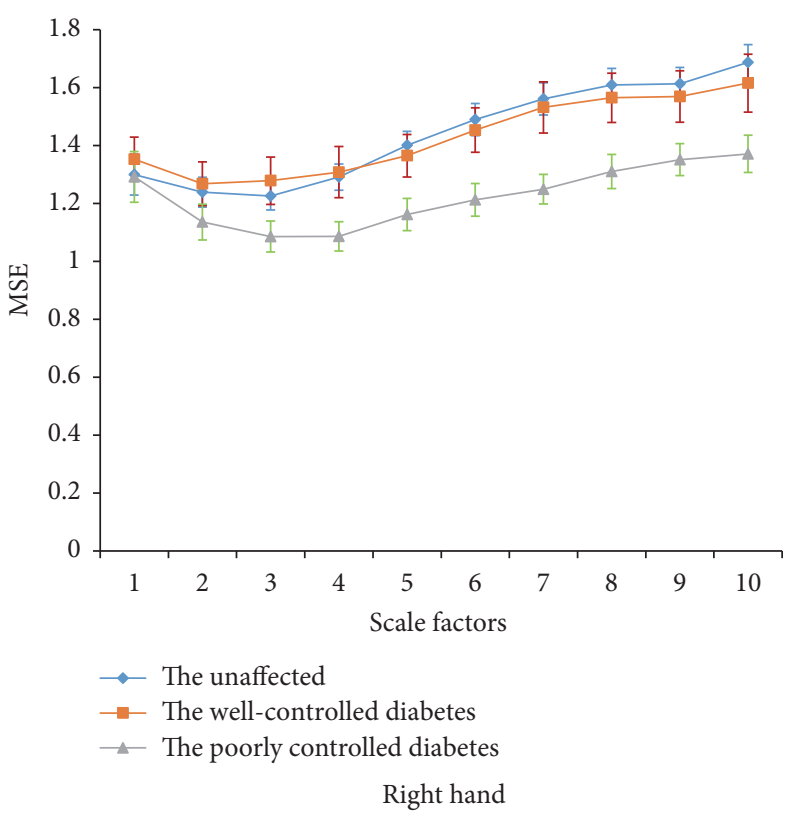

(a)

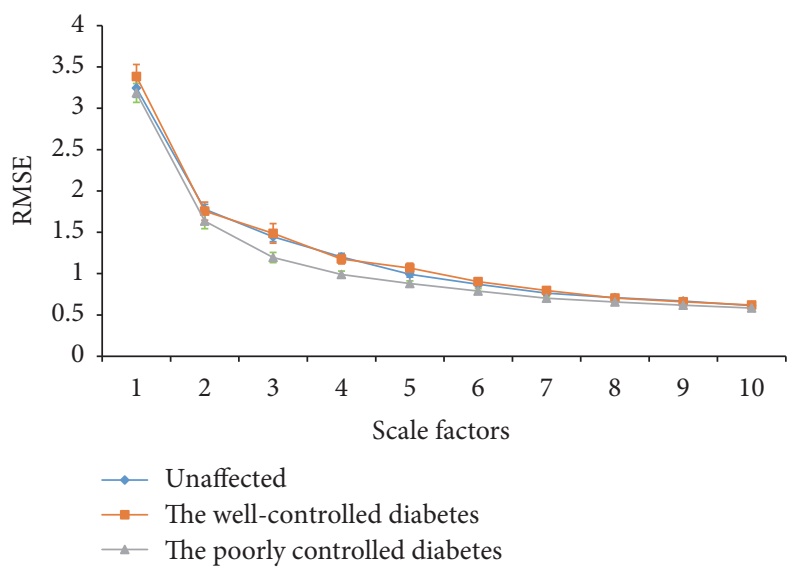

Right hand

(c)

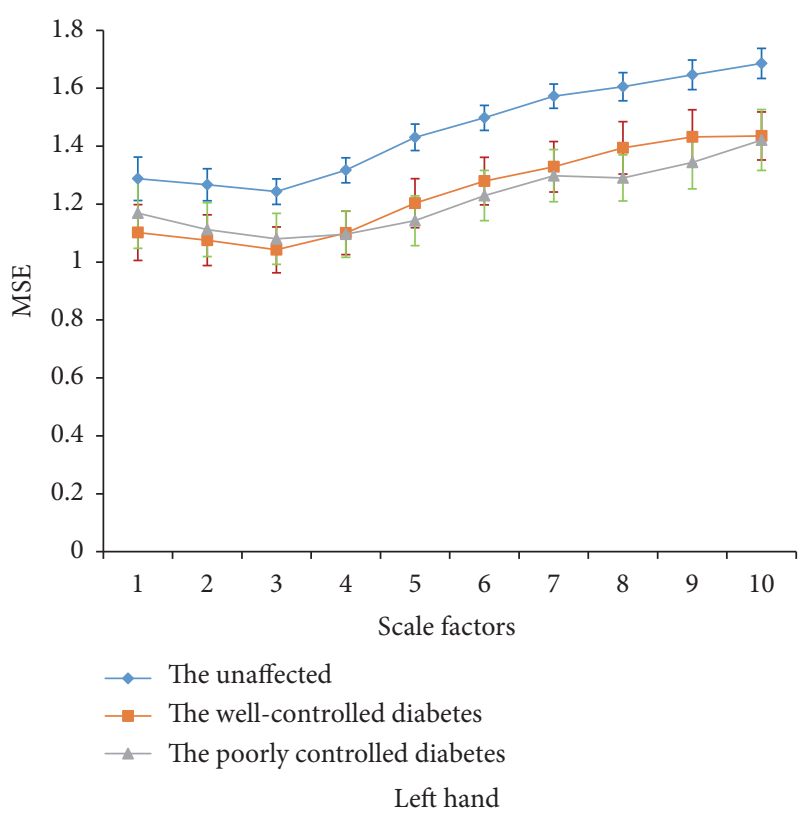

(b)

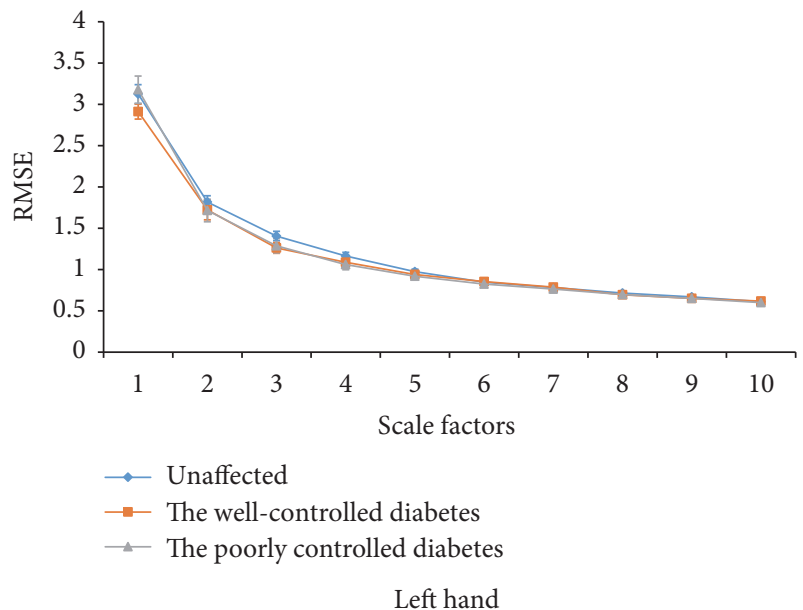

(d)

FIgURE 2: The MSE and RMSE index with the standard error (vertical bar) of the unaffected (solid blue line), the well-controlled diabetes (solid orange line), and the poorly controlled diabetes (solid grey line) groups at each scale factor in left and right hands, respectively.

the influence of handgrip strength on any time scales MSE or RMSE was not remarkable in individuals without diabetes and those with poorly controlled diabetes.

Several physiological signals such as sleep electroencephalographic wave [26], electromyographic signals [27], body temperature [28], intracranial pressure [29], pulse rate [12], and blood pressure [15] have been used for MSE analysis to mainly evaluate autonomic nervous dysfunction, cardiovascular disease, treatment effect, and disease prognosis. Trunkvalterova et al. revealed that the MSE of blood pressures and pulse rate at scale factor $\tau=3$ in patients with type 1 diabetes were significantly lower than those in the unaffected controls [15]. In addition, the complexity of glucose dynamics evaluated by MSE was lower in type 1 or 2 diabetes than that in the healthy controls [30]. Previously, we used ECG R-R intervals and pulse transit times, the time interval between the R-wave peak of the ECG waveform to the footpoint of the PPG pulse from left toe, for MSE and multiscale crossapproximate entropy (MCAE) analyses in type 2 diabetes [20]. The results showed the MSE of ECG R-R intervals at scale 1 did not differ between the unaffected controls and those with diabetes. On the contrary, the large-scales MSE of pulse transit times, which was defined as the time interval between the appearance of ECG peak R-wave and the following PPG pulse amplitude, was lower in patients with diabetes. The MCAE as the irregularity between ECG and PPG signals further distinguished patients with wellcontrolled diabetes from those with poorly controlled diabetes. Moreover, we also uncovered the large-scales MCAE between ECG R-R intervals and PPG pulse amplitudes of left index finger decreased in the same manner in type 2 diabetes [31]. 


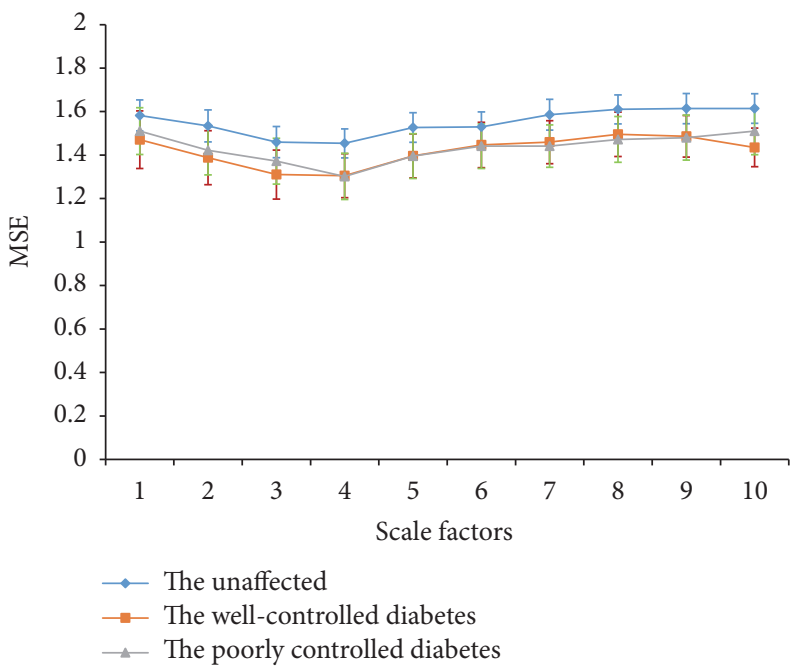

(a)

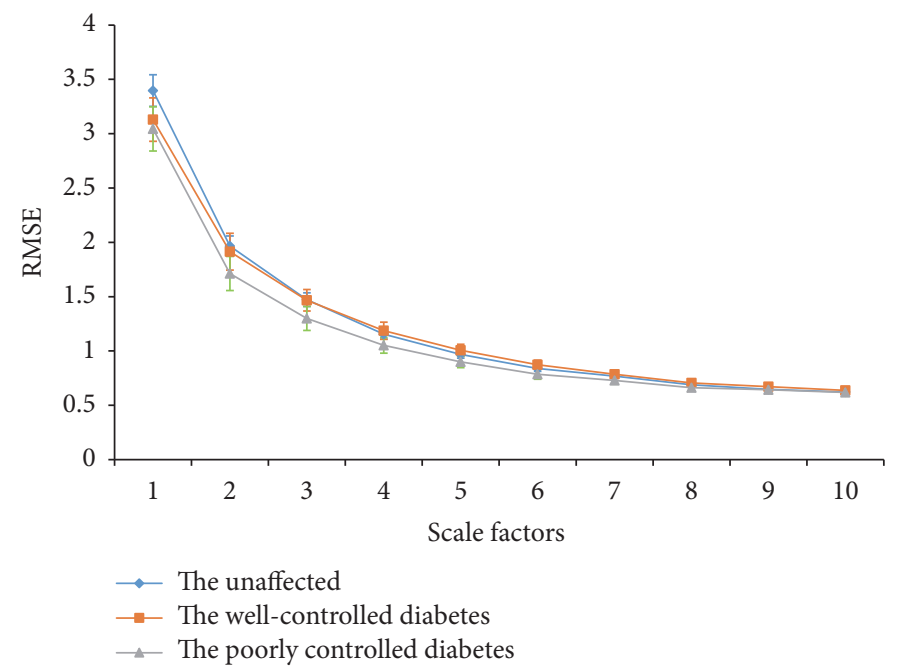

(b)

Figure 3: The MSE and RMSE index of ECG R-R intervals with the standard error (vertical bar) of the unaffected (solid blue line), the well-controlled diabetes (solid orange line), and the poorly controlled diabetes (solid grey line) groups at each scale factor.

Several studies have shown that as compared with those free of diabetes, diabetic patients with cardiac or peripheral autonomic neuropathy related to poor glycemic control and sedentary status have lower baroreflex sensitivity [32, 33]. The impaired short-term cardiovascular control in diabetes mellitus may be caused by the functional or structural impairment of nerve fibers of the autonomic nervous system innervating the heart and blood vessels [34]. de MouraTonello et al. [35] further concluded that the presence of diabetic neuropathies may account for the additional impairment of the sympathetic control to the blood vessels, possibly leading to the reduction of large-scale MSE, while the vagal impairment is a primary result of type 1 or 2 diabetes which is associated with the reduced small-scale MSE [36].

Our study firstly clarified that peripheral microvascular dysfunction and autonomic neuropathy in type 2 diabetes evaluated by small-, medium-, and large-scales MSE and RMSE might be related to glycemic control and hand dominance. We noticed that in absence of peripheral vascular disease (e.g., subclavian artery subtotal occlusion), the mean PPG pulse amplitudes and cardiofinger PWV did not differ between two hands in all the groups. The dominant hand, having more local physical activity than the nondominant hand, may improve regional microvascular circulation, thereby reducing the risk of peripheral neuropathy and increasing the small-scale $\mathrm{MSE}_{1}$ or RMSE $\mathrm{R}_{1}$ in patients with type 2 diabetes. However, if blood glucose was not controlled well, the peripheral neuropathy remained present in dominant hand. These findings were consistent with many previous observational studies $[3-9,37]$.

To the best of our knowledge, PPG pulse amplitudes are closely related to central or peripheral autonomic nervous, cardiorespiratory, and vascular controls [17], whereas ECG $\mathrm{R}-\mathrm{R}$ intervals, the classic biological signals mostly used for the entropy analysis, are mainly influenced by autonomic nervous activity. Since the PPG pulse amplitudes are compromised by a wide range of physiological functions, the complexity of PPG signal series might be more sensitive than heart rate variability as a short-term estimate of autonomic and vasomotor dysfunction in diabetes. Furthermore, the complexity analyses of PPG pulse amplitudes could give an opportunity to independently evaluate peripheral vascular health of each distal extremity.

The strengths of our study include the availability of large numbers of ECG R-R intervals and serial PPG pulse amplitude signals of bilateral fingertips and the availability of a wide range of data on other covariates. Additionally, we used case-control comparisons to lessen the effect of age, sex, and weight on the MSE and RMSE. In contrast, our study was cross-sectional in nature and the laboratory data such as HbAlc levels were measured once that we could not avoid the effect of remote glycemic controls on the results. Finally, residual confounding may have attributed to the difference between groups.

We concluded that increased physical activity of local extremities and blood glucose controlled at HbAlc levels $<8 \%$ are equally vital to prevent diabetic microvascular complications. In addition, simultaneous measurements of PPG pulse amplitudes signals of bilateral fingers rather than HRV for MSE or RMSE analysis might be clinically useful to evaluate diabetic neuropathy and microvascular dysfunction.

\section{Conflicts of Interest}

The authors declare that they have no conflicts of interest.

\section{Authors' Contributions}

Hsien-Tsai Wu and Gen-Min Lin contributed equally to the work. 


\section{Acknowledgments}

This work was supported by the research grants from the Ministry of Science and Technology, Taiwan (Grant nos. MOST 104-2221-E-259-014 and MOST 105-2221-E259-007).

\section{References}

[1] K. Yoda, M. Inaba, K. Hamamoto et al., "Association between glycemic control and morning blood pressure surge with vascular endothelial dysfunction in type 2 diabetic patients," Diabetes Care, vol. 37, no. 3, pp. 644-650, 2014.

[2] I. Vinagre, J. L. Sánchez-Quesada, J. Sánchez-Hernández et al., "Inflammatory biomarkers in type 2 diabetic patients: effect of glycemic control and impact of LDL subfraction phenotype," Cardiovascular Diabetology, vol. 13, article 34, 2014.

[3] R. Klein, B. E. Klein, S. E. Moss, and K. J. Cruickshanks, "Relationship of hyperglycemia to the long-term incidence and progression of diabetic retinopathy," Archives of Internal Medicine, vol. 154, pp. 2169-2178, 1994.

[4] L. D. Bash, E. Selvin, M. Steffes, J. Coresh, and B. C. Astor, "Poor glycemic control in diabetes and the risk of incident chronic kidney disease even in the absence of albuminuria and retinopathy: Atherosclerosis Risk in Communities (ARIC) study," Archives of Internal Medicine, vol. 168, no. 22, pp. 24402447, 2008.

[5] R. J. Young, D. J. Ewing, and B. F. Clarke, "Nerve function and metabolic control in teenage diabetics," Diabetes, vol. 32, no. 2, pp. 142-147, 1983.

[6] B. Hemmingsen, S. S. Lund, C. Gluud et al., "Targeting intensive glycaemic control versus targeting conventional glycaemic control for type 2 diabetes mellitus," The Cochrane database of systematic reviews, vol. 11, no. CD008143, 2013.

[7] A. Maiorana, G. O’Driscoll, C. Cheetham et al., “The effect of combined aerobic and resistance exercise training on vascular function in type 2 diabetes," Journal of the American College of Cardiology, vol. 38, no. 3, pp. 860-866, 2001.

[8] G. Fuchsjäger-Mayrl, J. Pleiner, G. F. Wiesinger et al., "Exercise training improves vascular endothelial function in patients with type 1 diabetes," Diabetes Care, vol. 25, no. 10, pp. 1795-1801, 2002.

[9] S. Balducci, G. Iacobellis, L. Parisi et al., "Exercise training can modify the natural history of diabetic peripheral neuropathy," Journal of Diabetes and Its Complications, vol. 20, no. 4, pp. 216223, 2006.

[10] A. I. Vinik, R. E. Maser, B. D. Mitchell, and R. Freeman, "Diabetic autonomic neuropathy," Diabetes Care, vol. 26, no. 5, pp. 1553-1579, 2003.

[11] M. Javorka, Z. Trunkvalterova, I. Tonhajzerova, J. Javorkova, K. Javorka, and M. Baumert, "Short-term heart rate complexity is reduced in patients with type 1 diabetes mellitus," Clinical Neurophysiology, vol. 119, no. 5, pp. 1071-1081, 2008.

[12] M. Costa, A. L. Goldberger, and C.-K. Peng, "Multiscale entropy analysis of complex physiologic time series," Physical Review Letters, vol. 89, no. 6, article 068102, 2002.

[13] L. E. V. Silva, R. M. Lataro, J. A. Castania et al., "Nonlinearities of heart rate variability in animal models of impaired cardiac control: contribution of different time scales," Journal of Applied Physiology, vol. 123, no. 2, pp. 344-351, 2017.

[14] M. Costa, A. L. Goldberger, and C.-K. Peng, "Multiscale entropy analysis of biological signals," Physical Review E: Covering
Statistical, Nonlinear, Biological, And Soft Matter Physics, vol. 71, no. 2, Article ID 021906, 2005.

[15] Z. Trunkvalterova, M. Javorka, I. Tonhajzerova et al., "Reduced short-term complexity of heart rate and blood pressure dynamics in patients with diabetes mellitus type 1: multiscale entropy analysis," Physiological Measurement, vol. 29, no. 7, pp. 817-828, 2008.

[16] H. T. Wu, P. C. Hsu, C. F. Lin et al., "Multiscale entropy analysis of pulse wave velocity for assessing atherosclerosis in the aged and diabetic," IEEE Transactions on Biomedical Engineering, vol. 58, no. 10, pp. 2978-2981, 2011.

[17] M. Elgendi, "On the analysis of fingertip photoplethysmogram signals," Current Cardiology Reviews, vol. 8, no. 1, pp. 14-25, 2012.

[18] G.-M. Lin, B. Haryadi, C.-M. Yang, S.-C. Chu, C.-C. Yang, and H.-T. Wu, "Discrepancies between conventional multiscale entropy and modified short-time multiscale entropy of photoplethysmographic pulse signals in middle- and old- aged individuals with or without diabetes," Entropy, vol. 19, no. 3, article 132, 2017.

[19] H.-T. Wu, C.-C. Yang, G.-M. Lin et al., "Multiscale crossapproximate entropy analysis of bilateral fingertips photoplethysmographic pulse amplitudes among middle-to-old aged individuals with or without type 2 diabetes," Entropy, vol. 19, no. 4, article 145, 2017.

[20] H.-T. Wu, C.-C. Liu, M.-T. Lo et al., "Multiscale crossapproximate entropy analysis as a measure of complexity among the aged and diabetic," Computational and Mathematical Methods in Medicine, vol. 2013, Article ID 324325, 7 pages, 2013.

[21] Z. Wu, N. E. Huang, S. R. Long, and C. Peng, "On the trend, detrending, and variability of nonlinear and nonstationary time series," Proceedings of the National Academy of Sciences of the United States of America, vol. 104, no. 38, pp. 14889-14894, 2007.

[22] N. E. Huang, Z. Shen, S. R. Long et al., "The empirical mode decomposition and the Hilbert spectrum for nonlinear and non-stationary time series analysis," The Royal Society of London. Proceedings. Series A. Mathematical, Physical and Engineering Sciences, vol. 454, no. 1971, pp. 903-995, 1998.

[23] J. S. Richman and J. R. Moorman, "Physiological time-series analysis using approximate entropy and sample entropy," American Journal of Physiology-Heart and Circulatory Physiology, vol. 278, no. 6, pp. H2039-H2049, 2000.

[24] V. Bari, J. F. Valencia, M. Vallverdú et al., "Multiscale complexity analysis of the cardiac control identifies asymptomatic and symptomatic patients in long QT syndrome type 1," PLoS ONE, vol. 9, no. 4, Article ID e93808, 2014.

[25] J. F. Valencia, A. Porta, M. Vallverdú et al., "Refined multiscale entropy: application to 24-h holter recordings of heart period variability in healthy and aortic stenosis subjects," IEEE Transactions on Biomedical Engineering, vol. 56, no. 9, pp. 2202-2213, 2009.

[26] I. R. Bell, A. Howerter, N. Jackson, M. Aickin, R. R. Bootzin, and A. J. Brooks, "Nonlinear dynamical systems effects of homeopathic remedies on multiscale entropy and correlation dimension of slow wave sleep EEG in young adults with histories of coffee-induced insomnia," Homeopathy, vol. 101, no. 3, pp. 182-192, 2012.

[27] H.-T. Wu, Y.-H. Jiang, A.-B. Liu et al., "Multiscale entropy analysis of surface electromyographic signals from the urethral sphincter as a prognostic indicator for surgical candidates with primary bladder neck obstruction," Entropy, vol. 17, no. 12, pp. 8089-8098, 2015. 
[28] V. E. Papaioannou, I. G. Chouvarda, N. K. Maglaveras, G. I. Baltopoulos, and I. A. Pneumatikos, "Temperature multiscale entropy analysis: a promising marker for early prediction of mortality in septic patients," Physiological Measurement, vol. 34, no. 11, pp. 1449-1466, 2013.

[29] C. W. Lu, M. Czosnyka, J. S. Shieh, A. Smielewska, J. D. Pickard, and P. Smielewski, "Complexity of intracranial pressure correlates with outcome after traumatic brain injury," Brain, vol. 135, no. 8, pp. 2399-2408, 2012.

[30] J.-L. Chen, P.-F. Chen, and H.-M. Wang, "Decreased complexity of glucose dynamics in diabetes: evidence from multiscale entropy analysis of continuous glucose monitoring system data," American Journal of Physiology-Regulatory Integrative and Comparative Physiology, vol. 307, no. 2, pp. R179-R183, 2014.

[31] H.-T. Wu, C.-Y. Lee, C.-C. Liu, and A.-B. Liu, "Multiscale crossapproximate entropy analysis as a measurement of complexity between ECG R-R interval and PPG pulse amplitude series among the normal and diabetic subjects," Computational and Mathematical Methods in Medicine, vol. 2013, Article ID 231762, 7 pages, 2013.

[32] A. Frattola, G. Parati, P. Gamba et al., "Time and frequency domain estimates of spontaneous baroreflex sensitivity provide early detection of autonomic dysfunction in diabetes mellitus," Diabetologia, vol. 40, no. 12, pp. 1470-1475, 1997.

[33] J. Tank, A. Neuke, A. Mölle, J. Jordan, and M. Weck, "Spontaneous baroreflex sensitivity and heart rate variability are not superior to classic autonomic testing in older patients with type 2 diabetes," American Journal of the Medical Sciences, vol. 322, no. 1, pp. 24-30, 2001.

[34] A. I. Vinik and D. Ziegler, "Diabetic cardiovascular autonomic neuropathy," Circulation, vol. 115, no. 3, pp. 387-397, 2007.

[35] S. C. de Moura-Tonello, A. Porta, A. Marchi et al., "Cardiovascular variability analysis and baroreflex estimation in patients with type 2 diabetes in absence of any manifest neuropathy," PLoS ONE, vol. 11, no. 3, article e0148903, 2016.

[36] S. C. de Moura-Tonello, A. C. Takahashi, C. O. Francisco et al., "Influence of type 2 diabetes on symbolic analysis and complexity of heart rate variability in men," Diabetology and Metabolic Syndrome, vol. 6, article no. 13, 2014.

[37] P. M. Kluding, M. Pasnoor, R. Singh et al., "The effect of exercise on neuropathic symptoms, nerve function, and cutaneous innervation in people with diabetic peripheral neuropathy," Journal of Diabetes and Its Complications, vol. 26, no. 5, pp. 424429, 2012. 


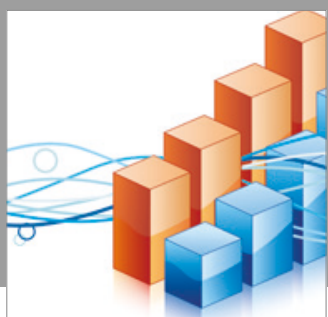

Advances in

Operations Research

vatersals

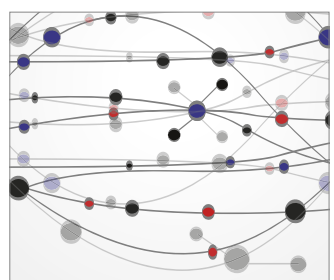

\section{The Scientific} World Journal
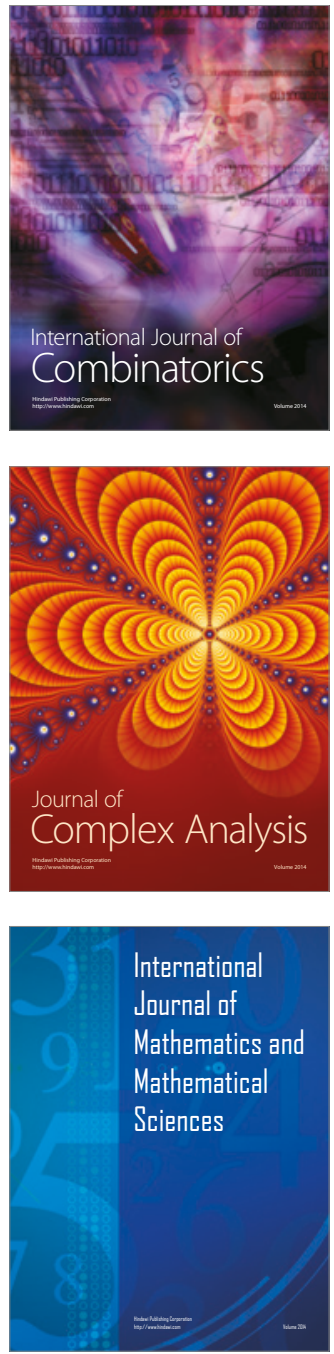
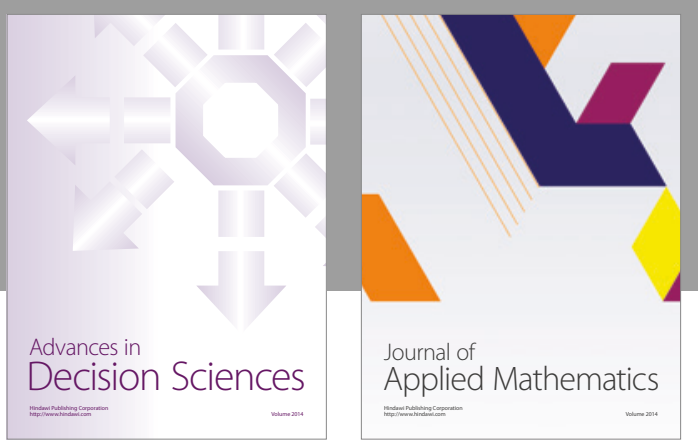

Algebra

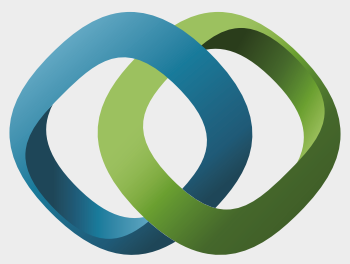

\section{Hindawi}

Submit your manuscripts at

https://www.hindawi.com
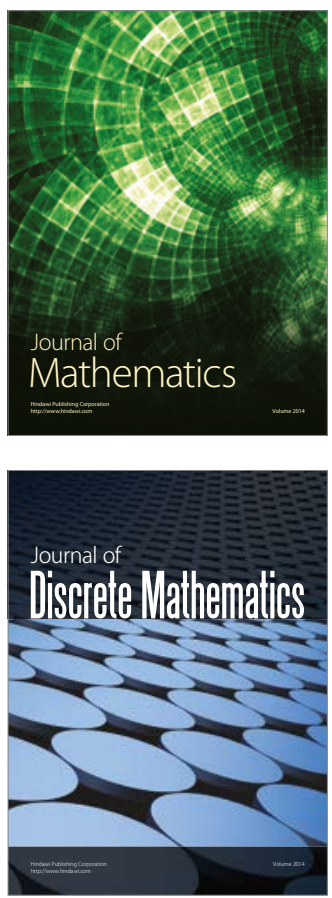

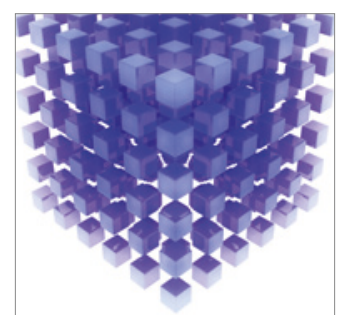

Mathematical Problems in Engineering
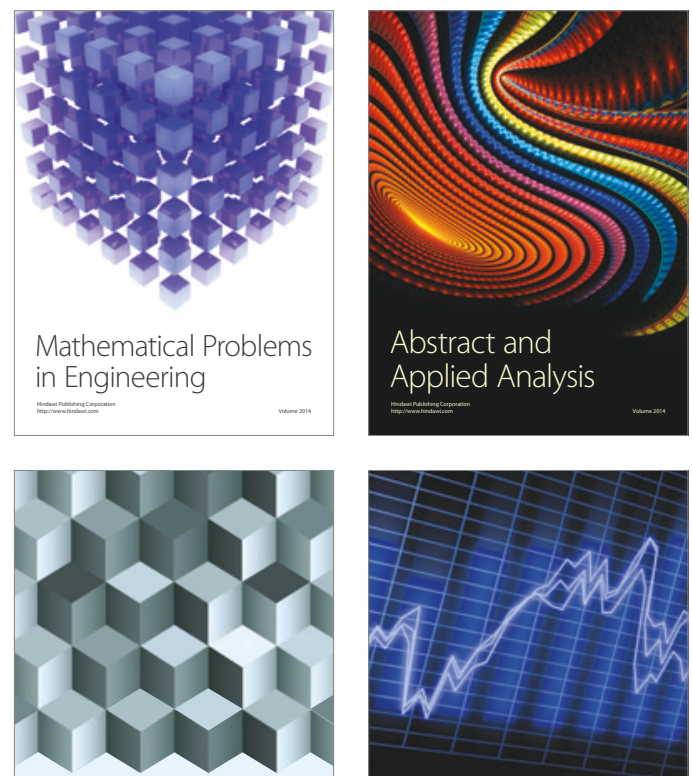

Journal of

Function Spaces

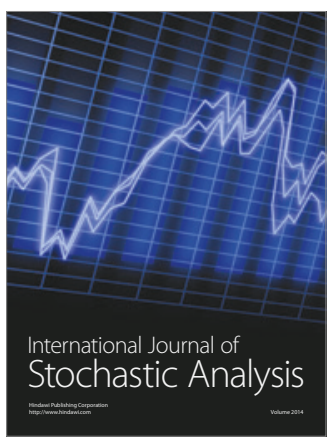

Probability and Statistics
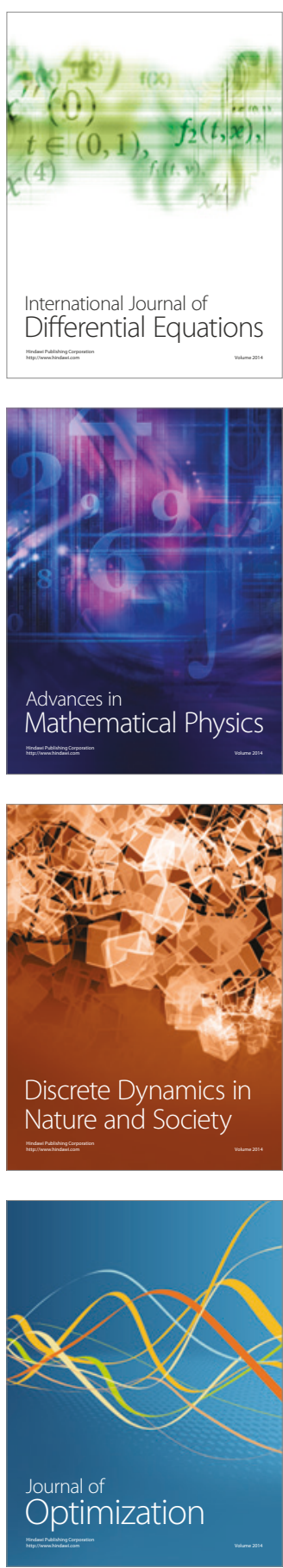